\title{
Chemical activities of graphitic carbon spheres
}

\author{
Z.C. Kang, Z.L. Wang * \\ School of Material Science and Engineering, Georgia Institute of Technology, Atlanta, GA 30332-0245, USA
}

Received 22 April 1996; accepted 9 September 1996

\begin{abstract}
Monodispersive carbon spheres with a high percentage of purity have been synthesized using a mixed-valent oxide-catalytic carbonization (MVOCC) process. In this paper, the chemical activities of the carbon spheres are examined by dispersing $\mathrm{Pt}, \mathrm{CdS}$ and $\mathrm{WO}_{3}$ nanoparticles, respectively, on the carbon surfaces from the microstructure information provided by transmission electron microscopy. The spheres are composed of graphitic flakes with high density open edges at the surfaces, thus, they are a good candidate for catalysis applications.
\end{abstract}

Keywords: Carbon sphere; $\mathrm{Pt}$; $\mathrm{CdS} ; \mathrm{WO}_{3}$; Graphitic flake

\section{Introduction}

Carbon is a versatile element because it can form various structures with unique properties and applications [1,2]. Carbon is also an important catalyst, see many articles in Refs. $[3,4]$. The surface chemical activity of carbon is closely related to its microstructure because the reactivity of carbon is determined by the density of open graphitic edges at the surface. Carbon black (or soot) is a widely used material in industry, but it refers to a specific group of carbon particles defined to be a small spheroidally shaped, paracrystalline, non-dis-

\footnotetext{
* Corresponding author. E-mail: zhong.wang@mse.gatech.edu.
}

crete component of an aggregate that can be separable from the aggregate only by fracturing [5], such as the particles observed in Refs. [6,7].

Recently, we have introduced a mixed-valent oxide-catalytic carbonization (MVOCC) process for synthesizing monodispersive carbon spheres (or tubes) in macroscopic quantities at low cost and with high purity product $[5,6]$. The spheres are composed of graphitic flakes and are believed to exhibit many dangling bonds at the surfaces. These spheres have distinguishable differences from the carbon black particles. In this paper, the surface chemical activities of the newly synthesized carbon spheres are demonstrated through their interactions with $\mathrm{Pt}, \mathrm{CdS}$ and $\mathrm{WO}_{3}$ nanoparticles. The porosity and reaction sites created by $\mathrm{WO}_{3}$ at the carbon surfaces are observed using high-resolution transmission 
electron microscopy (HRTEM), and the physical mechanism which drives this interaction process is illustrated. It is concluded that the carbon spheres are an excellent candidate for catalysis or as a support for catalysts.

\section{Synthesis of carbon spheres}

Carbon spheres with an average diameter of 210-600 nm were synthesized using the MVOCC process [8]. Transition-metal oxides and/or rare earth metal oxides with mixed-valences are the catalysts. The MVOCC process is similar to the classical gas decomposition process under heating, but the key difference is the oxide catalyst. The carbon electrode arc-discharging and the gas decomposition techniques reported in the literature both use metal particles, such as $\mathrm{Fe}, \mathrm{Ni}, \mathrm{Cu}$, transition metals or rare earth metals as catalysts. These catalysts not only promote the carbonization of the gas vapor but also serve as the nucleation sites for carbon products [10]. Thus, the catalysts are capsulated after carbonization, and the purification of the final product is a major problem. The metal oxides with mixed valences used in MVOCC overcome this difficulty, and more technical details have been given elsewhere $[8,9]$.

To study the surface chemical activity of these spheres, nanoparticles and the carbon spheres were mixed and ultrasonically dispersed in ethanol for $10 \mathrm{~min}$. Many particles were adhered on the surfaces of the spheres, which were then picked up by a holey carbon grid for TEM observation. The HRTEM experiments were performed at $400 \mathrm{kV}$ using an JEOL $4000 \mathrm{EX}$ TEM, which routinely gives a $0.18 \mathrm{~nm}$ structure image resolution.

\section{Experimental results}

The as-synthesized carbon spheres were first examined by scanning electron microscopy
(SEM). The carbon spheres shown in Fig. 1 have an average diameter of $600 \mathrm{~nm}$. The full width at half maximum of the size distribution is $50 \mathrm{~nm}$. The product is pure and clean, and the smallest average size of the spheres synthesized by MVOCC is $210 \mathrm{~nm}$. Chemical microanalysis using energy dispersed X-ray spectroscopy (EDS), at a detection limit of $1 \%$, has found no other elements but carbon.

Fig. 2a is a low-magnification TEM image of the as-synthesized spheres, which clearly shows a spherical shape. The carbon spheres are dispersive and solid. An HRTEM image recorded near the edge of a carbon sphere shows the graphitic structure of the sphere (Fig. 2b). The graphitic flakes are not closed shells but small waving flakes of atom-thickness, ranging in sizes from 1-10 $\mathrm{nm}$. The arrangement of the graphitic flakes follows the curvature of the sphere at different depths. The interplanar distance is modulated by the waving structure of the flakes, and its average value is approximately $0.34 \mathrm{~nm}$.

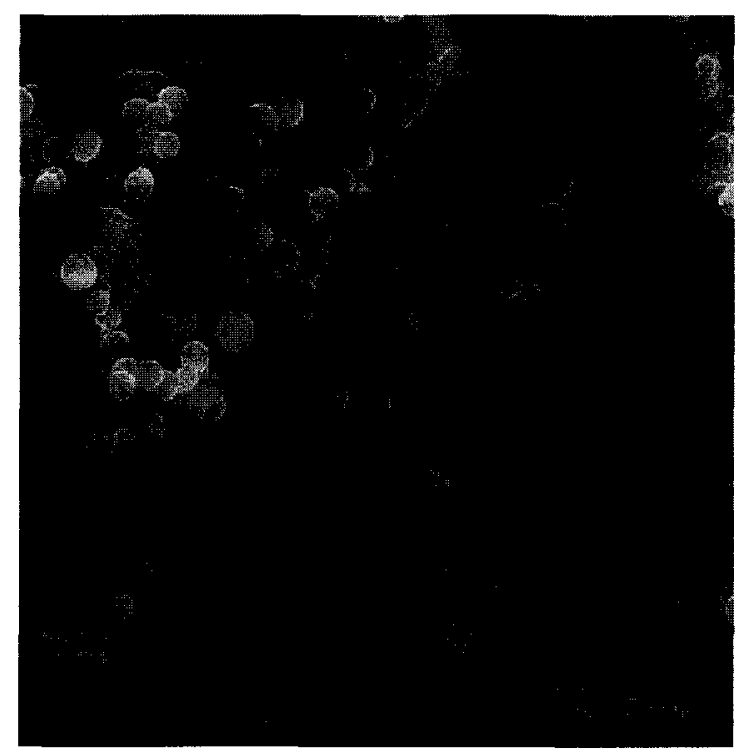

Fig. 1. An SEM image of carbon spheres synthesized using the MVOCC process. The product is pure and no carbon tubes or any other product was observed. The image was recorded at $15 \mathrm{kV}$ using a Hitachi S800 SEM. 
The unclosed graphitic flakes create many open edges at the surface, which are highly desired for stimulating surface reactions. These open edges have been shown as the key microstructure that is responsible for the accretion between spheres after a ultrasonic treatment in acetone [11]. A growth model has been proposed for these spheres [8]. The spheres are extremely stable in air and at high temperature and they are resistive to oxidation and corrosion. We now show the interaction between the carbon spheres with several different types of nanoparticles.

\subsection{Pt / C}

Our first experiment is to examine the interaction of platinum particles with the surfaces of carbon spheres. The Pt nanoparticles were prepared using the method of Rampino and Nord [12] and Henglein et al. [13] by reduction of $\mathrm{K}_{2} \mathrm{PtCl}_{4}$ using $\mathrm{Ar}$ and $\mathrm{H}_{2}$ gases in the presence of polyacrylate solution, and details have been reported elsewhere [14]. The $\mathrm{Pt}$ particles have facet shape with $\{111\}$ and $\{100\}$ planes, with a size distribution of $3-20 \mathrm{~nm}$, as indicated by the HRTEM image shown in Fig. 3a. The particle is

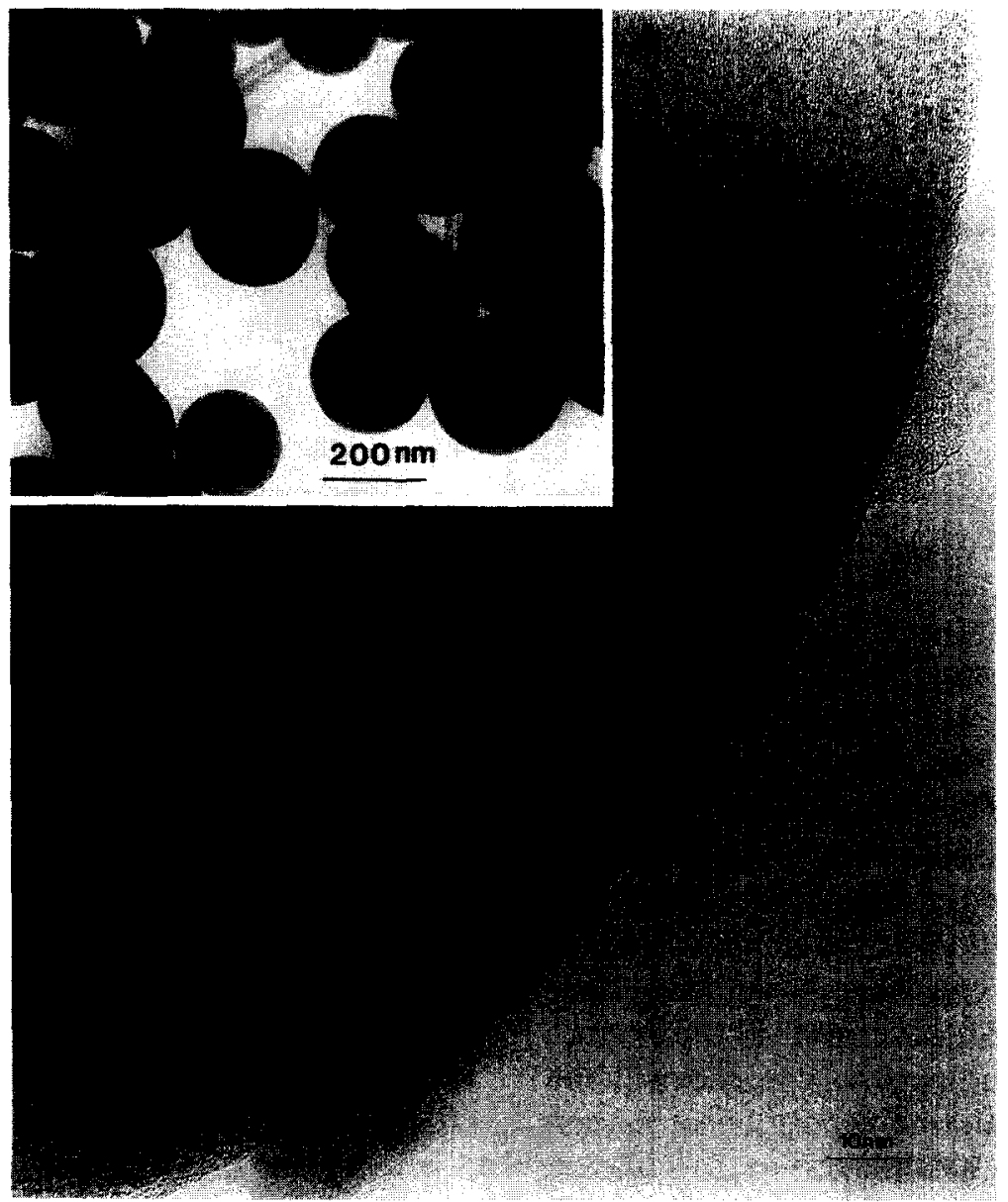

Fig. 2. (a) A low-magnification TEM image of the synthesized carbon spheres by the MVOCC process (b) $\Lambda$ n HRTEM image recorded from an edge of a carbon sphere, showing waving graphitic structures. The species in darker contrast on the sphere surface are dispersed $\mathrm{WO}_{3}$ particles for the studies to be discussed in Section 3.3. 


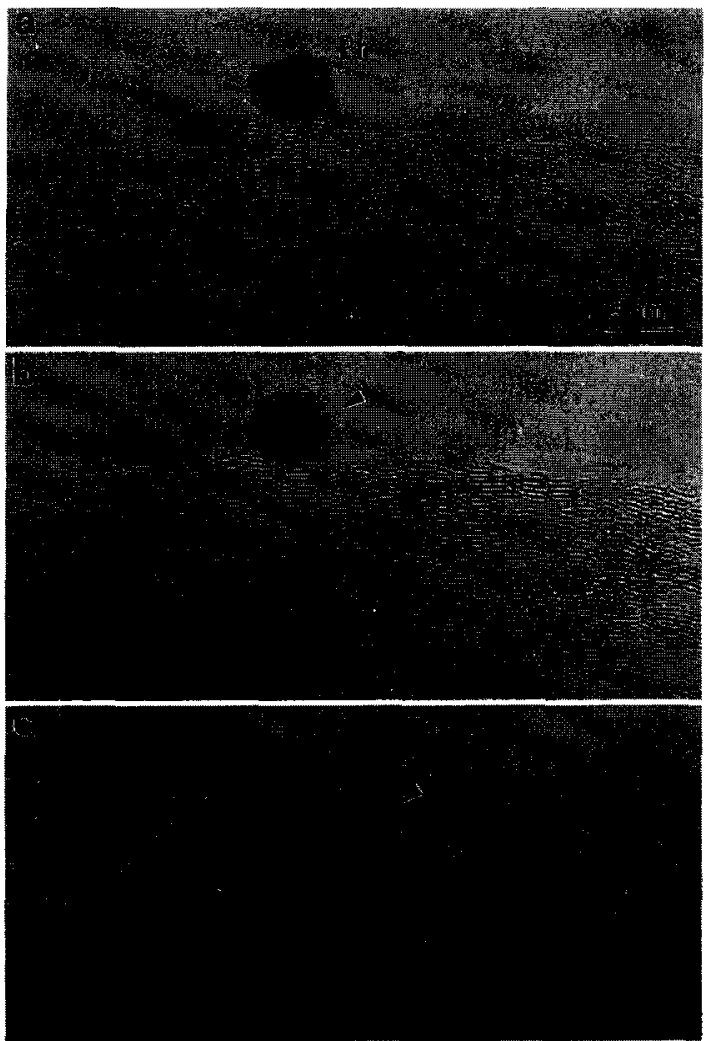

Fig. 3. A time series of TEM images recorded from a Pt particle adhered on the carbon surface at an interval of 10 min, showing the interaction of the particle with the sphere under the stimulation of the electron beam.

adhered on the surface. After a few minutes under the electron beam, some carbon species are formed onto the Pt surface (as indicated by an arrowhead in Fig. 3b), but no significant evolution in particle shape is found. After about $10 \mathrm{~min}$ of intensive beam illumination, the particle orientation is rotated and many open-ends graphitic edges are created at the carbon surface (indicated in Fig. 3c). The over all microstructure is that the Pt particle does not react with the carbon sphere, while the deposition of carbon on the Pt surface is possible due to the spillover process, indicating that the carbon surface is effective for supplying hydrogen and oxygen adsorbed on carbon surface.

\section{2. $C d S / C$}

Semiconductor nanoparticles have attracted much interests because of the prospective quantum dot effect. CdS is such a typical example. The CdS particles used in our studies were prepared using the method of Mews et al. [15]. When first dispersed on the carbon sphere surface, $\mathrm{CdS}$ particles are not perfectly crystalline (Fig. 4a). After being illuminated by the electron beam for a few minutes, the crystallinity of the CdS particles is significantly improved, and many fine particles as small as $2-5 \mathrm{~nm}$ are observed (Fig. 4b). In comparison to the image recorded first under the electron beam, the carbon surface is strongly attacked and it appears that the CdS particles are enclosed by carbon. An aggregate of $\mathrm{CdS}$ particles on the carbon surface has reacted with carbon based on its structure evolution in Fig. 4a, b. The facet shape of the CdS particles and the porosity of the carbon surface created by the interaction with CdS are apparent in regions near the large aggregate (Fig. 4c). The most frequently observed facets of CdS are $\{111\}$.

\section{3. $\mathrm{WO}_{3} / \mathrm{C}$}

Nanosize $\mathrm{WO}_{3}$ particles are dispersed on the surfaces of the carbon spheres in ethanol. A low-magnification TEM image recorded from a carbon sphere clearly indicates the decoration of small $\mathrm{WO}_{3}$ clusters, in sizes as small as $0.5 \mathrm{~nm}$, uniformly on the carbon surface (Fig. 5a). The $\mathrm{WO}_{3}$ used here was commercial powders ground in a molar pestle. The particles are very fine and the average distance between the adjacent clusters is approximately $3 \mathrm{~nm}$. For a carbon sphere of $200 \mathrm{~nm}$ in diameter, there are more than $10,000 \mathrm{WO}_{3}$ clusters decorated on its surface. These small size clusters are believed to be formed in the dissolution process of $\mathrm{WO}_{3}$ in ethanol, and they tend to distribute at the sites where are the open graphitic edges on the sur- 
face of the carbon sphere. The adhesion of $\mathrm{WO}_{3}$ clusters on the surface greatly changes the structure of the carbon spheres. Fig. 5b shows an HRTEM image at an edge of a carbon sphere, where many $\mathrm{WO}_{3}$ clusters are seen and the carbon surface is strongly attacked, resulting in high surface porosity. The 'neck' type structure is formed between the sphere and the particle and many open graphitic edges are created, thus, the surface area is greatly increased. This is useful in catalysis.

\section{Discussion}

The high surface activity of the carbon spheres is directly associated with the graphitic structure of the spheres. We now examine the bonding in different carbon structures to illustrate this point. The bonding in diamond is dominated by the $\mathrm{sp}^{3}$ electronic configuration, the four valence electrons of a carbon atom are all in the three-dimensional tetrahedral oriented $\mathrm{sp}^{3}$ hybrid orbital, forming strong $\mathrm{s}$ bonding
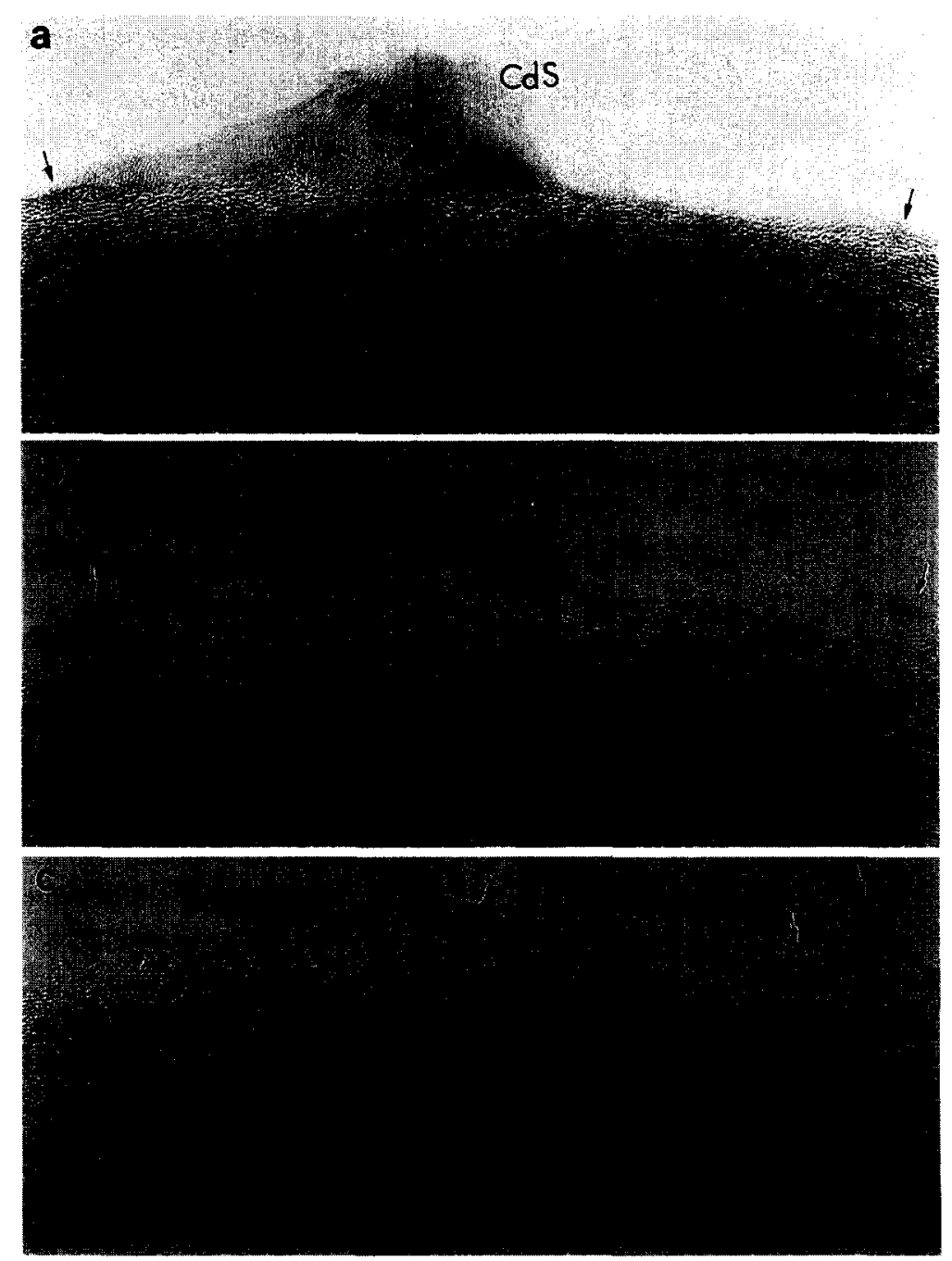

Fig. 4. (a) and (b) are TEM images of CdS particles adhered on the carbon surface showing the structural evolution due to the interaction of $\mathrm{CdS}$ with carbon surface. (c) shows another region of the sphere with the formation of small size faceted particles. 

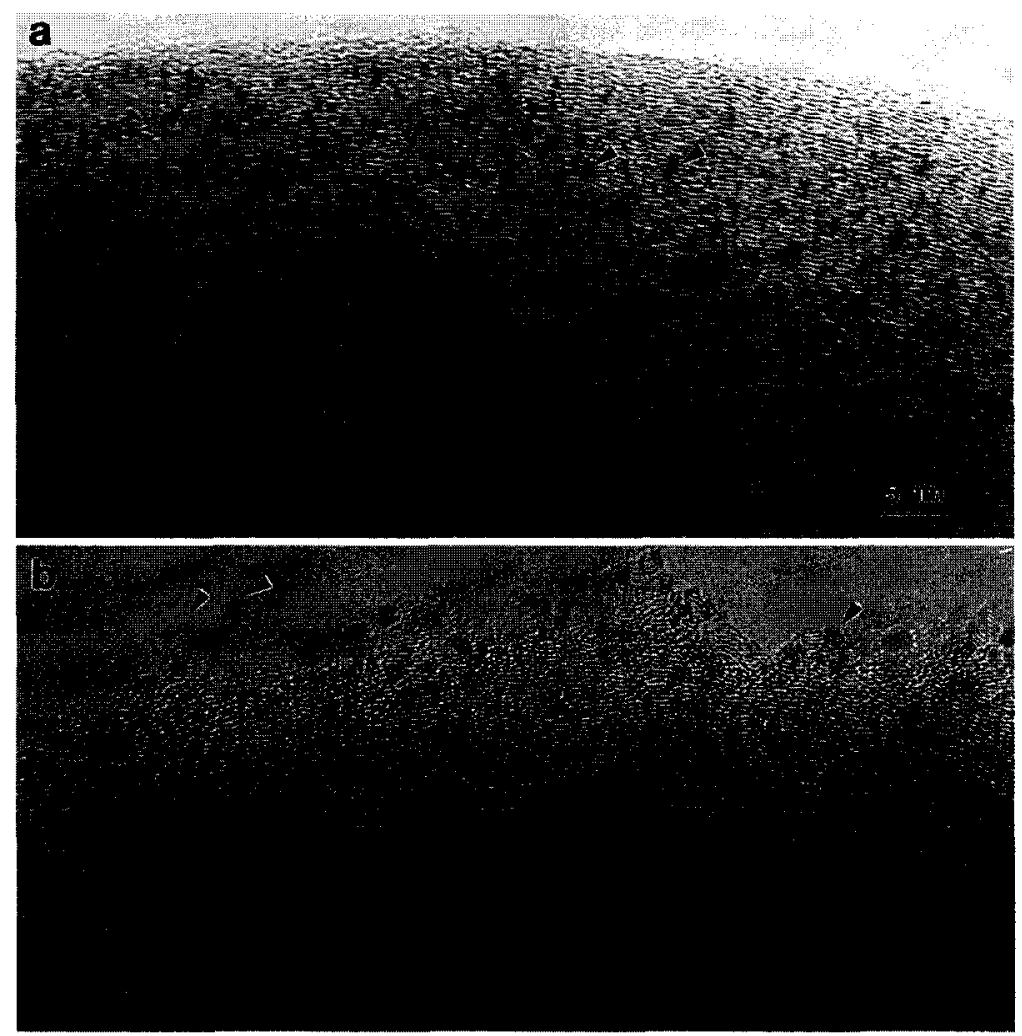

Fig. 5. TEM images of carbon spheres recorded at a time interval of 30 min showing the attack of carbon surface by $\mathrm{small}^{\mathrm{WO}_{3}}$ clusters under the electron beam, resulting in the formation of the neck structure beneath the particles, as indicated by arrowheads.

with the adjacent atoms [1]. These bonds are probably the most strongest ones in crystalline materials and they are unlikely to be opened, thus, no chemical reaction is possible. For graphite, the 3 -fold coordinated $\mathrm{sp}^{2}$ electronic configuration places three out of four valence electrons in a two-dimensional trigonally oriented $\mathrm{sp}^{2}$ hybrid orbital, forming strong intralayers bonding within the hexagonal carbonrings; the fourth electron is in the $\mathrm{p} \pi$ orbital which is perpendicular to the hexagonal network, forming a weakly unsaturated $\pi$ bonding with adjacent layers. Thus, chemical reaction is possible only at the open edges of the graphite layers because of the unbalanced dangling bonds.

Although the discovery of $\mathrm{C}_{60}$ has made a great impact on the understanding of carbon bonding, the chemical reactivity of $\mathrm{C}_{60}$ might be limited for the following reason. For a $\mathrm{C}_{60}$, 12 pentagonal carbon-rings must be introduced to form a close cage structure with 20 hexagonal carbon-rings. Each atom in $\mathrm{C}_{60}$ has three adjacent neighbors although the atoms may form pentagonal carbon-rings. Thus, the nearest neighbor distribution in $\mathrm{C}_{60}$ is approximately equivalent to the atom distribution in a hexagonal graphite layer, and the electronic configuration can still be described by the $\mathrm{sp}^{2}$ hybrid orbital although some distortion may exist due to the atom distribution in the $\mathrm{C}_{60}$ cage. The fourth unsaturated $\pi$ bonding electron would like to form a pair with an electron from another $\mathrm{C}_{60}$, forming the weakly bonded $\mathrm{C}_{60}$ crystals. Moreover, the $\mathrm{C}_{60}$ cage is unlikely to be opened by an external electron due to the weakness of the unsaturated $\mathrm{p}$ bonding normal to the surface. Therefore, $\mathrm{C}_{60}$ is considered to be chemically 
inert. Similarly, carbon spheres with closed shell structures [16] are expected to have analogous chemical characteristics as $\mathrm{C}_{60}$.

However, the situation is very different if the graphitic layers composing the carbon spheres are unclosed, leaving many dangling bonds at the open sites. This is just the structure of the newly synthesized carbon spheres. The experimental observations in Section 3 have demonstrated that the carbon surface is most strongly attacked by $\mathrm{WO}_{3}$, while $\mathrm{Pt}$ is least reactive with carbon. The moderate chemical activity of semiconductor $\mathrm{CdS}$ is probably determined by the surface adsorbed species on the carbon sphere, where the unpaired $\mathrm{sp}^{2}$ orbital is usually balanced by the adsorbed hydrogen, oxygen and/or $\mathrm{OH}$ [17]. A combination of $\mathrm{S}$ atoms with those adsorbed atoms may result in surface reaction. This process occurs only for the surface adsorbed species and no carbon atoms are expected to take part.

The interaction of $\mathrm{WO}_{3}$ with carbon surface directly involve carbon, and it can be interpreted using an internal Auger decay mechanism proposed by Feibelman and Knoteck [18] for the electron stimulated desorption of $\mathrm{WO}_{3}$. Under the excitation of an electron beam, one electron in the W $4 \mathrm{f}$ or $\mathrm{W} 5 \mathrm{p}$ state is ionized, then, one electron from $\mathrm{O} 2 \mathrm{p}$ fills the hole created by the ionized $\mathrm{W}$ electron; the energy released from this down transition is sufficient to ionize two electrons belonging to $\mathrm{O} 2 \mathrm{p}$. Thus, $\mathrm{O}^{2-}$ loses up to three electrons and is converted into a positively charged ion $\mathrm{O}^{+}$; the Coulomb repulsion between $\mathrm{O}^{+}$and an $\mathrm{W}$ ion results in the desorption of $\mathrm{O}^{+}$, and $\mathrm{WO}_{3}$ is reduced to W. Since an $\mathrm{O}^{+}$ion located beneath the surface layer is unlikely to escape, this desorption process occurs only to the atoms located at the surface. The desorbed $\mathrm{O}^{+}$ions tend to be attracted by the carbon atoms located at the edges of the open graphitic layers on the carbon surface. The combination of $\mathrm{O}$ with $\mathrm{C}$ produces gas phase $\mathrm{CO}_{2}$ or $\mathrm{CO}$, resulting in the loss of local carbon and the formation of surface porosity. The reduced $\mathrm{W}$ atoms at the surface may tend to diffuse toward the carbon sphere; the aggregation of the $\mathrm{W}$ atoms forms the observed clusters (Fig. 5). This process is likely to occur for atoms diffused from the $\mathrm{WO}_{3}$ surface, thus the reaction occurs at the vicinity of the $\mathrm{WO}_{3}$ particle rather than at the contact region between $\mathrm{WO}_{3}$ and the carbon sphere because the $\mathrm{O}$ ions located inside the contact region cannot escape, resulting in the formation of the neck structure observed in Fig. 5b [19].

It is known that the carbon surface is lipophilic and oxides are hydrophilic, the system composed of dispersed oxides (e.g., $\mathrm{WO}_{3}$ ) on carbon may exhibit both characteristics. Thus, the carbon spheres may be a potential catalyst for synthesizing enzymes which contain lipophilic and hydrophilic components.

The HRTEM image in Fig. 2b clearly shows that the carbon surface has high density graphitic edges, on which hydrogen, oxygen, $\mathrm{OH}$ and many organic function groups, such as lactone, phenol and carbonyl, are expected to be adsorbed. The deposited nanoparticles (e.g., Pt, $\mathrm{CdS}$ and $\mathrm{WO}_{3}$ ) on the carbon surface form a composite catalyst with these groups, which may be used to promote organic reactions.

The experimental results presented in this paper unavoidably involve the effect of electron beam. In some cases, the reaction energy is provided by the electron beam due to the local heating effect. In other cases, the electron beam stimulated ionization is necessary. Although we cannot revoke the beam effect, these data have shown the experimental feasibility and potential applications of the carbon spheres.

\section{Conclusions}

The newly synthesized carbon spheres exhibit high surface chemical activities. The interactions between $\mathrm{Pt}, \mathrm{CdS}$ and $\mathrm{WO}_{3}$ with the carbon surface suggest that electron transfer is possible in these systems. The interactions (observed under the electron beam) produce high surface porosity and reaction sites, thus, the 
carbon spheres can be used for catalysis or as a support for catalysts. The strength of the interaction depends strongly on the type of the materials. For semiconductor CdS, the surface adsorbed species on carbon are believed as the sources of reaction. For oxides, such as $\mathrm{WO}_{3}$, the interaction mechanism is likely due to the reduction of $\mathrm{W}$ from $\mathrm{WO}_{3}$ as a result of an internal Auger decay process under the stimulation of an electron beam in TEM.

\section{Acknowledgements}

Thanks to Dr. T.S. Ahmadi for kindly providing the Pt nanoparticles.

\section{References}

[1] J. Robertson, Prog. Solid State Chem. 21 (1991) 199.

[2] C.N.R. Rao, R. Seshadri, A. Govindaraj and R. Sen, Mater. Sci. Eng. R 15 (1995) 209.
[3] R. Burch (Ed.), Carbon and Catalysis, Catal. Today 7(2) (1990).

[4] A.J. Bird, in: Catalyst Supports and Supported Catalysts (Butterworth Publishers, Stoneham, 1987) p. 107.

[5] W.M. Hess and C.R. Herd, in: J.-B. Donnet, R. C. Bansal and M.-J. Wang (Eds.), Carbon Black Science and Technology, 2nd Ed. (Marcel Dekker Inc., New York, 1993) pp. 106-108.

[6] R. Richter, K. Hernadi, R. Caudano, A. Fonseca, H.N. Migeon, J.B. Nagy, S. Schneider, J. Vandooren and P.J. Van Tiggelen, Carbon 34 (1996) 427.

[7] C.M. Megaridis and R.A. Dobbins, Combust. Sci. Technol. 66 (1989) 1.

[8] Z.C. Kang and Z.L. Wang, Philos. Mag. B 73 (1996) 905.

[9] Z.L. Wang and Z.C. Kang, Philos. Mag. B 74 (1996) 51.

[10] S. Amelinckx, X.B. Zhang, D. Bernaerts, X.F. Zhang, V. Ivanov and J.B. Nagy, Science 265 (1994) 635.

[11] Z.C. Kang and Z.L. Wang, J. Phys. Chem. 100 (1996) 5163.

[12] L.D. Rampino and F.F. Nord, J. Am. Chem. Soc. 63 (1942) 2745.

[13] A. Henglein, B.G. Ershov and M. Marlow, J. Phys. Chem. 99 (1995) 14129.

[14] T.S. Ahmadi, Z.L. Wang, T.C. Green, A. Henglein and M.A. El-Sayed, Science 272 (1996) 1924

[15] A. Mews, A. Eychmüller, M. Giersig, D. Schooss and H. Weller, J. Phys. Chem. 98 (1994) 934.

[16] D. Ugarte, Nature 359 (1992) 707.

[17] T.J. Fabish and J. Schleifer, Carbon 22 (1984) 19.

[18] P.J. Feibelman and M.L. Knotek, Phys. Rev. 18 (1978) 6531.

[19] Z.L. Wang and Z.C. Kang, Carbon (1996), in press. 